


\section{Comisión Editorial}

Mercedes Mesén

Consultora externa. San José, Costa Rica; mmesen@gmail.com

- Ida Fallas

Escuela de Ciencias de la Educación, UNED, San José, Costa Rica; ifallas@uned.ac.cr

Alí Víquez

UNED, San José, Costa Rica; aviquez@uned.ac.cr

Lady Meléndez

Escuela de Ciencias de la Educación, UNED, San José, Costa Rica; Imelendez@uned.ac.cr

\section{Irma Zúñiga}

Consultora externa. San José, Costa Rica; irmazunigaleon@gmail.com

Luis Paulino Vargas

Vicerrectoría de Investigación, UNED, San José, Costa Rica; Ivargas@uned.ac.cr

Antonio Bartolomé

Universidad de Barcelona; abartolome@ub.edu

- Karl Steffens

Universidad de Colonia, Alemania; Karl.Steffens@uni-koeln.de

\section{Eugenia Chaves}

Escuela de Ciencias de la Educación, Sistema de Estudios de Posgrado, UNED, San José, Costa Rica; echaves@uned.ac.cr

Innovaciones Educativas es una publicación académica anual de la Universidad Estatal a Distancia (UNED).

Las opiniones en la revista no son necesariamente avaladas por la UNED. La responsabilidad compete a los autores.

Servicios de información: Eugenia Chaves

Editora

Apdo. 474-2050, San Pedro de Montes de Oca, Costa Rica, C.A.

echaves@uned.ac.cr

Editor Gráfico: Sergio Aguilar Mora

Diseño Gráfico y Diagramación: Mónica Chávez Ramos

Coordinador de Producción Editorial: Daniel Villalobos Gamboa

Impreso en los Talleres Gráficos de la Editorial EUNED, San José, Costa Rica 


\section{Presentación}

esde esta tribuna, nos complace poner a disposición de la comunidad universitaria y del público interesado en la educación un conjunto de artículos con temáticas muy variadas. La riqueza de esta variedad se justifica en el marco de la educación a distancia y de los resultados de la aplicación de nuevas estrategias en la mediación pedagógica. El hilo conductor es la visión innovadora de nuestros autores para enfocar temas o escenarios de aprendizaje desde una óptica que potencie la transformación.

Presentamos un primer artículo de María Gabriela Marín y Lady Meléndez sobre Construyamos centros educativos inclusivos. Un modelo para evaluar actitudes, políticas y acciones institucionales. Pretende coadyuvar en la transición de las escuelas hacia la educación inclusiva, mediante la autoevaluación y el replanteamiento de las actitudes, políticas y acciones institucionales, a la luz del Índice de Inclusión de Ainscow y Booth (2000). Esta experiencia se realiza en tres centros costarricenses de educación primaria: Escuela José Figueres Ferrer, Escuela Granadilla Norte y Escuela Monterrey Vargas Araya, que hoy constituyen centros modelo de educación inclusiva. La experiencia se desarrolla como un proyecto del Programa de Educación Especial de la UNED.

Seguidamente, se presenta un análisis sobre la relación entre Prematuridad y estimulación temprana: ¿un binomio determinante para la prevención de la discapacidad?, de Jorge Baxter, Linda Madriz y Ana Lorena Mora. Se revisa y estudia una selección de investigaciones de México y Costa Rica que han discutido el vínculo entre ambos temas. Busca integrar y analizar sus resultados con el objetivo de dar sustento teórico a una nueva investigación interesada en explorar los mismos tópicos en ambos países; además, formular un protocolo de atención temprana en la prevención de la discapacidad en niños y niñas nacidos antes de tiempo y con factores de riesgo biológico asociados.

En un esfuerzo por ampliar nuestras fronteras y llegar a la comunidad con dominio del idioma inglés, presentamos un artículo sobre la hiperactividad con déficit atencional, titulado AttentionDeficit Hyperactivity Disorder in the EFL Classroom: A Case Study, de Olmedo Bula, en el cual se reflexiona sobre la experiencia de la puesta en práctica de un plan para un estudiante con trastorno de hiperactividad con déficit de atención, que asiste a un colegio en un entorno de ILE en Costa Rica. El estudio reporta las estrategias y técnicas usadas por el profesor para mejorar el desempeño del estudiante en el aula. Los resultados y el análisis de este estudio de caso son también abordados.

El siguiente artículo, de Julián Monge y Lizette Brenes, sugiere Cómo lograr una investigación de calidad internacional e impacto real en la Universidad Estatal a Distancia de Costa Rica. La UNED ha asumido el compromiso de fortalecer, ampliar y adaptar las líneas de investigación en las áreas de competencias básicas del aprendizaje, medios didácticos, cognición y lenguaje. Se presenta un estudio comparativo con las demás universidades costarricenses y se concluye con una 
propuesta de política institucional para: crear comunidades científicas interdisciplinarias o multidisciplinarias con al menos un investigador de impacto internacional; fomentar la movilidad de los investigadores y su interacción nacional e internacional; dar los recursos y estímulos necesarios para hacer investigación de impacto nacional e internacional en mayor escala; ayudar a la divulgación científica especializada y popular; flexibilizar a los estudiantes para que desarrollen competencias en investigación y en temas científicos innovadores, y crear mecanismos para la participación activa de los estudiantes en las comunidades científicas y en los proyectos.

La educación a distancia experimenta nuevos campos como el aprendizaje de segundas lenguas, de ahí la importancia del artículo Acerca de la función de lo estético-cognitivo en el proceso de lecto-escritura para la adquisición de segundas lenguas, de María Isabel Barboza. Mediante la respuesta del lector a textos literarios, desde un marco teórico bidireccional, estético-cognitivo, se puede observar la interacción y transacción entre el texto y el lector, a la vez que coadyuva al proceso de enseñanza-aprendizaje de una segunda lengua.

La formación para la investigación como eje curricular en la formación docente: el caso de la Carrera de Educación Preescolar de la UNED-Costa Rica, de Jensy Campos y Rosa María Hidalgo, presenta resultados de una evaluación curricular realizada en la carrera de Educación Preescolar. La valoración se centra en la formación para la investigación; se concluye que $62.2 \%$ de los cursos del plan de estudios analizado incluyen actividades investigativas como parte de las acciones académicas desarrolladas por los estudiantes.

En el ámbito de la presentación de experiencias en el campo de la tecnología, presentamos la Iniciativa Open Courseware. El caso de la Cátedra Tecnologías Informáticas para la Educación, UNED. Se realiza una descripción de la iniciativa de Software Educativo Abierto u Open Courseware $(\mathrm{OCW})$ en las instituciones de educación superior, la experiencia de la incorporación en el proyecto OCW de dos cursos de la Cátedra Tecnologías Informáticas para la Educación, los cuales se imparten dentro del plan de estudios del Programa de Informática Educativa de la Escuela de Ciencias de la Educación, de la Universidad Estatal a Distancia (UNED) de Costa Rica.

Cerramos, como ha sido tradición, con un artículo de interés institucional, en este número incursionamos en La UNED ante las interacciones sociales digitales: el caso de audiovisuales en línea. Los audiovisuales en línea ante la sociedad digital, los centros universitarios y, en particular, la UNED deben de vislumbrar las nuevas necesidades y posibilidades de integración de su labor, de manera activa y permanente con la cotidianidad de sus usuarios potenciales. La plataforma de audiovisuales es una primera aproximación a la apertura de estos espacios y un ejemplo del manejo del ser institucional virtual como herramienta para la integración de la población estudiantil a la labor académica.

Agradecemos a nuestros colaboradores su esfuerzo y empeño para compartir con nuestros lectores sus valiosas experiencias y dar a conocer sus experiencias profesionales.

A todos muchas gracias por su apoyo. 